\title{
Necrotizing myopathy as a paraneoplastic syndrome associated with renal cell carcinoma
}

\author{
E. Naert ${ }^{1}$, J. L. De Bleecker ${ }^{2}$, N. Lumen ${ }^{3}$, S. Rottey ${ }^{1}$ \\ ${ }^{1}$ Department of Medical Oncology, ${ }^{2}$ Department of Neurology, ${ }^{3}$ Department of Urology, Ghent University \\ Hospital, Ghent, Belgium
}

We report a 49-year-old patient with necrotizing myopathy and a right renal mass. After laparoscopic radical nephrectomy, a remission of myopathy was seen. Pathologic evaluation of the nephrectomy specimen revealed a clear cell renal cell carcinoma. Relapse of myopathy 6 months postoperatively coincided with the diagnosis of the appearance of liver metastatic disease. After initiation of treatment with an mTOR-inhibitor, myopathy became less active requiring smaller amounts of corticosteroids with a complete remission of myopathy after 3 months of systemic treatment for metastatic renal cell cancer.

Keywords: Carcinoma, Renal cell, Paraneoplastic syndrome, Cancer-associated myositis, Necrotizing myopathy

\section{Patient History}

A 49-year-old man without significant medical history presented in June 2010 to the emergency unit with a 4-month history of muscle pain and weakness, mainly involving the proximal muscles, accompanied by subfebrility. Over the last weeks, he had developed malaise with fatigue, loss of appetite, increased dyspnea and weight loss of $3 \mathrm{~kg}$.

At the time of hospital admission, his blood pressure was $120 / 80 \mathrm{mmHg}$, heart rate 76 beats per minute and body temperature $36^{\circ} \mathrm{C}$. Notable findings on physical examination included painful thighs with muscle weakness grade 4/5 using Medical Research Council scale.

Laboratory data showed normocytic anemia, leukocytosis with left shift, thrombocytosis, hypoalbuminemia, elevated transaminases, markedly elevated serum creatine kinase (1821 U/l, reference values 10-195 U/l) and lactate dehydrogenase levels (1439 U/1, reference values 321-462 U/1). Results of renal function testing and urinalysis were normal. Viral cytology for Epstein-Barr virus, cytomegalovirus, HIV, hepatitis $\mathrm{A}, \mathrm{B}$ and $\mathrm{C}$ was negative.

Ultrasound of the abdomen revealed a $4.6 \times 7.5 \mathrm{~cm}$ heterogeneous mass in the upper/mid portion of the right kidney without signs of hydronephrosis. Further exploration with contrast-enhanced computed tomography (CT) of the abdomen and thorax

Correspondence to: Dr Eline Naert, Department of Medical Oncology, Ghent University Hospital, Ghent, Belgium. Email: eline.naert@ugent.be confirmed a low-attenuated septated mass in the right kidney accompanied by enlarged lymph nodes in the mediastinum and both hili of the lungs (largest measured 3.9 on $2.3 \mathrm{~cm}$ ). The patient was transferred to the urology department to undergo a retroperitoneal laparoscopic right radical nephrectomy with intact specimen extraction. As there were no enlarged abdominal lymph nodes, lymphadenectomy was not performed (cN0). A biopsy of the right vastus medialis was performed because of clinical evidence of myositis.

Pathologic examination revealed a $5-\mathrm{cm}$ renal cell carcinoma (RCC), clear cell type, Fuhrman grade 2 with negative surgical margins (pT1b).

In a second intervention, mediastinoscopy was performed for biopsy of one of the subcarinal lymph nodes. Pathologic examination revealed no malignant involvement ( $\mathrm{pN} 0$ ).

Muscle biopsy revealed a diffuse fiber atrophy with signs of a mild necrotizing myopathy without significant inflammation (Fig. 1). At that time, electromyography was not performed. After administration of corticosteroids and abundant fluid administration, his muscle weakness improved and CK values decreased (Fig. 2). Corticosteroids were phased out over 6 months because of gastric inconvenience and maintained on $4 \mathrm{mg}$ alternate day.

During follow-up, the patient showed a new episode of muscle pain and weakness located at thighs and shoulders. Laboratory findings again showed a highly elevated serum creatine kinase 


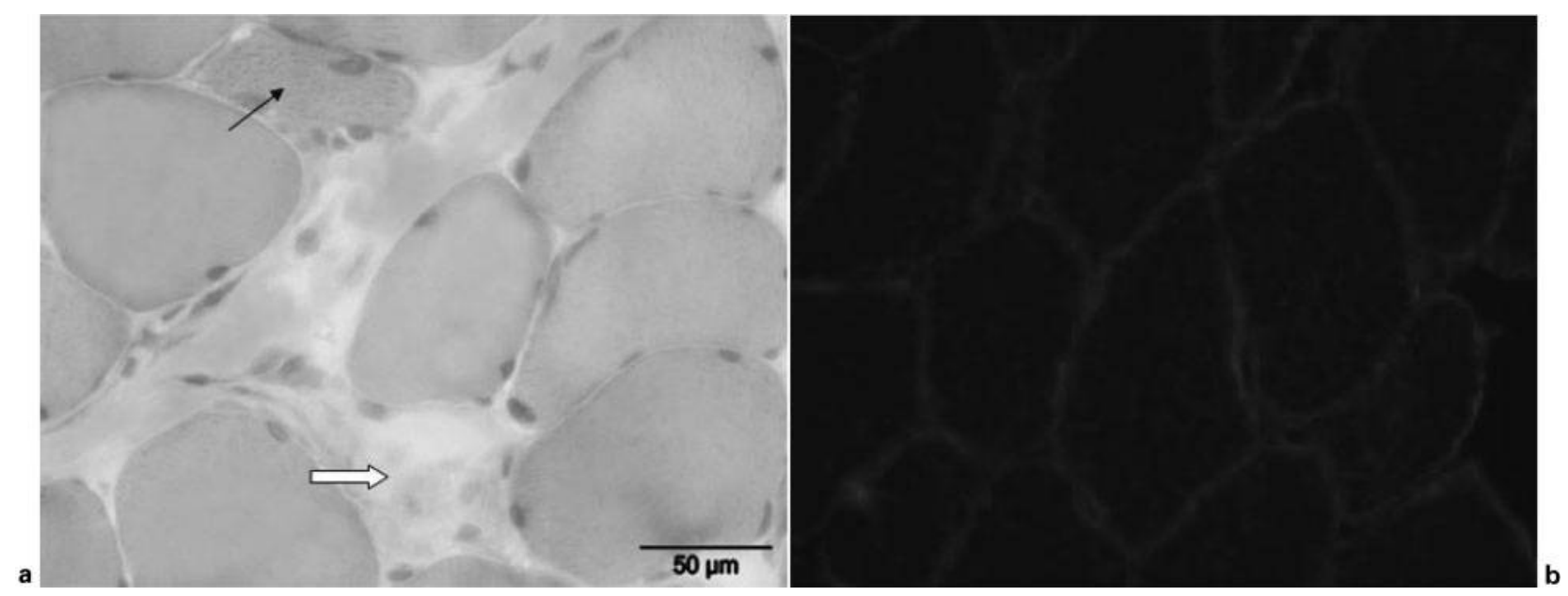

Figure 1 (A) Muscle biopsy showing a necrotic (large arrow) and a regenerating (small black arrow) muscle fiber indicating a mild necrotizing myopathy (modified trichrome stain, $\times 400$ ). (B) Immunofluorescence staining shows major histocompatibility class-I $(\mathrm{MHCl})$ reactivity on the sarcolemma of all muscle fibers $(\times 400)$

(10 $778 \mathrm{U} / \mathrm{l})$. Electromyography of the upper extremities revealed a myopathic process in the proximal muscles. A relapse of necrotizing myopathy was suggested and high dose steroid treatment $(64 \mathrm{mg}$ methylprednisolone) was restarted. A bone scan could not confirm bone involvement. Investigation with $\mathrm{CT}$ scan revealed a liver metastastic lesion in combination with bilateral enlarged lymph nodes at lung hili and mediastinum. After multidisciplinary discussion, the patient was diagnosed with metastatic renal cell carcinoma. Weekly administration of the mTOR (mammalian target of rapamycin) inhibitor temsirolimus was started (negative prognostic factors were elevated $\mathrm{LDH}$, anemia, and time from initial diagnosis to the start of systemic treatment $<1$ year) together with high dose steroids. Two months after start of administration, the metastatic lesion at the liver was in remission and serum creatine kinase normalized. There were no longer signs of muscle pain or weakness. Given the fact that both temsirolimus and steroids have an immunesuppressive effect, we tried to phase out the steroids as soon as possible (in about 8 months). Thirty-four months later, the patient is still receiving temsirolimus and CT scans demonstrate remaining remission of the disease according to RECIST criteria, without clinical signs of myositis and normal CK levels.

\section{Comment}

Our patient presented with clinical and serological abnormalities suggesting a myopathic process, pathologically classified as a necrotizing myopathy. There

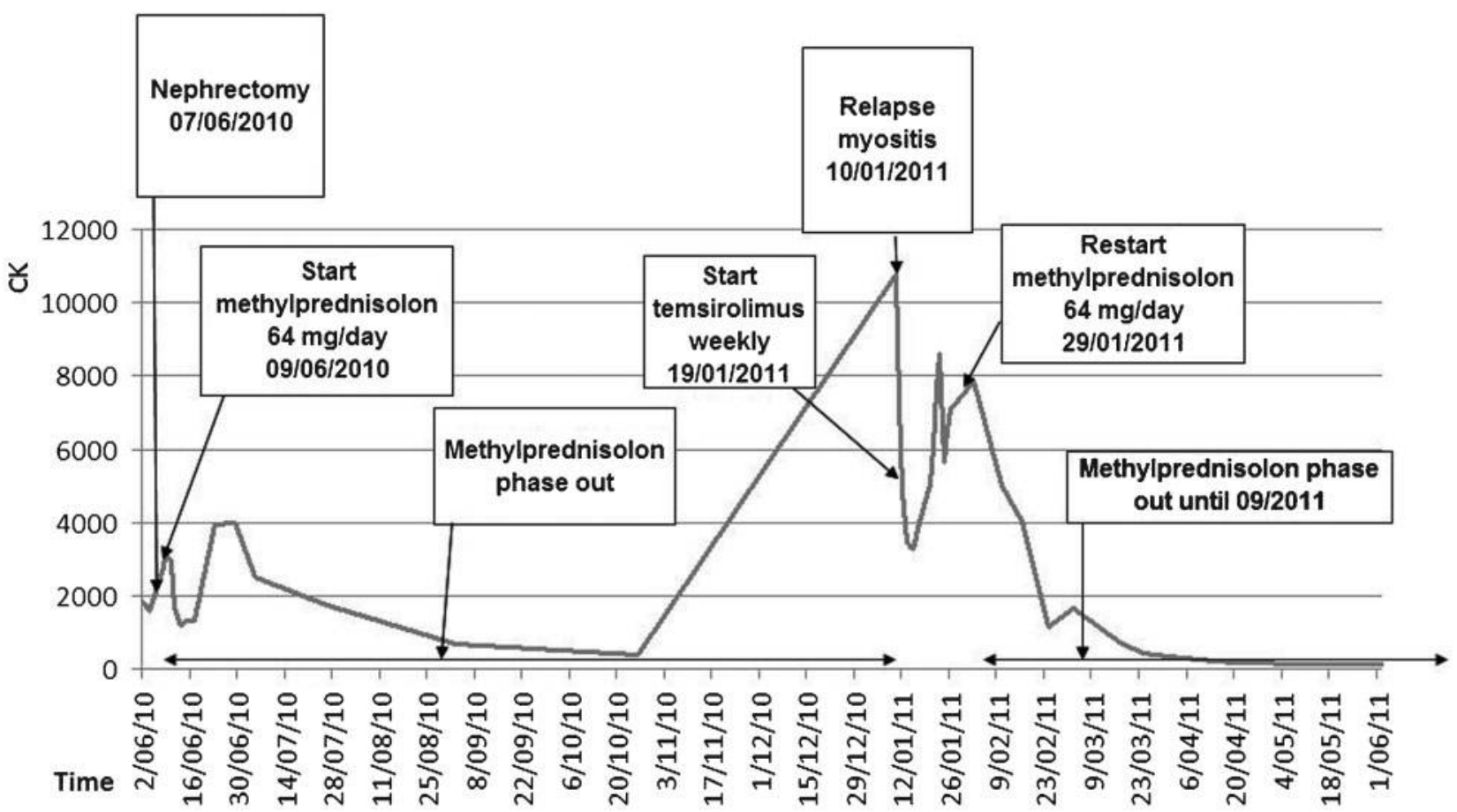

Figure 2 Evolution of serum creatine kinase during disease course 
was no medication, alcohol or substance abuse involved and there was no statin exposure. There were no anamnestic or clinical signs of systemic rheumatic diseases. Autoimmune serology (anti-CCP, ANCA, ANF, anti-mitochondrial antibody, anti-Sm, anti-Ro/SSA) was negative.

Necrotizing autoimmune myopathy (NAM) is a subtype of idiopathic inflammatory myopathies, a group of acquired, heterogeneous systemic diseases that mainly affect the skeletal muscles. The other three subgroups of idiopathic inflammatory myopathies are dermatomyositis, polymyositis and sporadic inclusion body myositis. In 2003 Anthony Amato on behalf of the muscle study group proposed the first formal definition of NAM: onset over 18 years, subacute or insidious onset of symmetric proximal more than distal limb and neck flexor more than neck extensor weakness, elevated serum CK levels and e.g. detection of myositis-specific antibodies (MSAs). ${ }^{1}$

This means a lot of non-specific myositis may have been falsely classified as dermatomyositis or polymyositis in the past. NAM is clinically resembling polymyositis, but histologically distinct by showing necrotic muscle fibres with minimal or absent inflammatory cell infiltration besides macrophages invading necrotic muscle fibers. ${ }^{2,3}$

Case reports and small series of patients reveal a subset of $16 \%$ of NAM characterized by anti-signal recognition particle (anti-SRP) antibodies. Although it seems to be an MSA, the link between these antibodies and myositis is not clear yet. Striking is the presence of additional symptoms like dysphagia or cardiac involvement and a higher CK level in SRPassociated myopathy., 3

Recent data showed a typical association of the anti3-hydroxy-3-methylglutaryl-coenzyme A reductase (antiHMGCR) antibody and NAM in statin-exposed patients. However, some patients with dermatomyositis, polymyositis and sporadic inclusion body myositis showed these antibodies too and on the other hand there were anti-HMGCR-positive patients that were not previously exposed to statins.,

Furthermore, NAM can occur in association with connective tissue diseases (anti-synthetase antibodies). About one-third of NAM is not accompanied by autoantibodies but may be associated with other autoimmune diseases or cancer. Neither anti-SRP nor anti-HMGCR antibodies were linked to cancer. Because of small series of patients reliable prevalence data for the four subgroups are not yet available. Neither SRP testing nor HMGCR testing was performed in our patient because commercial testing of the autoantibodies is however not yet available in Belgium. $^{3,4}$

As we detected a renal cell carcinoma, the pathologically proven necrotizing myopathy is very likely to be a cancer-associated myositis (CAM). NAM associated with cancer is minute giving the fact that a rapid positive evolution after short courses of steroids is seen. However, NAM associated with antiHMGCR and anti-SRP antibodies is known for mostly being slowly reactive to high dose steroids and to relapse frequently after tapering or stopping of steroids. $^{4}$

Approximately $20 \%$ of patients diagnosed with renal cell carcinoma present with a concomitant paraneoplastic syndrome. Another 10 to $40 \%$ of patients develop paraneoplastic symptoms during the disease. $^{5}$

Large epidemiologic studies confirmed the association of inflammatory myopathy with cancer. The strongest association is reported for dermatomyositis with standardized incidence ratios (SIRs) of 3.8-7.7. Large studies do show a greater risk of developing cancer for patients with polymyositis compared with cancer incidence rate in the general population (SIRs 1.7-2.0). Moreover, studies report that most cancers develop within two years of the onset of myositis, although the risk remains increased to 5 years after diagnosis. ${ }^{6}$

Dermatomyositis is strongly associated with ovarian, lung, gastric, colorectal and pancreatic cancers. In patients with polymyositis there was an increased risk of non-Hodgkin lymphoma, lung and bladder cancers. $^{3,6,7}$ The association between cancer and NAM has been reported, but to a much lesser extent compared with dermatomyositis and polymyositis. NAM affects merely adults over the age of 40, frequently with adenocarcinoma. ${ }^{8}$ SIR calculation has not yet been done due to the paucity of cases. ${ }^{2,3,6}$ No epidemiological data on the relation between CAM and renal cell carcinoma are available.

Screening for underlying malignancy in patients with newly diagnosed inflammatory myopathy is mandatory although there is no consensus on how extensive screening for associated cancer should be. ${ }^{3,6,7}$

The exact pathomechanism of NAM and CAM remains elusive. There is evidence that the presence of malignancy and immune-mediated destruction of muscle or skin is strongly linked in a way that tumor cells and injured and regenerating muscle cells seem to possess potential antigenic similarities. The tumor may thus be an antigen source in myositis and immune response also targets the injured and regenerating muscle cells that express the autoantigens. ${ }^{7,9}$

This positive correlation between tumor immunity and autoimmunity needs to be further explored.

There seems to be a correlation between the clinical course of myositis and malignancy, as successful treatment of the neoplasm will mostly impact on the muscle symptoms. Importantly, serum CK levels do 
not always correlate with the severity of weakness and secondly the severity of necrotizing myopathy does not always parallel tumor progression. ${ }^{3,7,8,10}$

Approximately 40 to $50 \%$ of patients with idiopathic autoimmune myopathy present MSA. The role of these MSA such as anti-Jo-1, anti-Mi-2, anti-SRP, anti-MJ and anti-p155, and myositis-associated-autoantibodies (MAA, present in autoimmune diseases with or without myositis) such as anti-PM-Scl, anti-Ku, anti-U1-RNP and anti-U3-RNP, in patients with myositis to predict CAM is currently a hot topic. In patients with paraneoplastic NAM these MSA and MAA can be either absent, non-specific of only weakly elevated. $3,6,7$

CAM has a worse prognosis compared to nonCAM with statistically significant higher mortality rates in the CAM group. Moreover, death in CAM almost always results from progression of malignant disease and not myositis, even in cases where the myositis is poorly controlled. ${ }^{7,10}$

In addition, the timely recognition of myositis onset is therapeutically important and contributes to morbidity and mortality: in patients where cancer and dermatomyositis are simultaneously diagnosed, the disease course is more severe than for those where the tumor is diagnosed prior to the myositis. It is still unclear whether or not we can extrapolate these data to the NAM subgroup. ${ }^{3,7,8}$

No studies are available that investigate morbidity and mortality in renal cell cancer versus renal cell cancer with CAM.

Based on case reports, we know that treatment of the tumor (surgery, chemotherapy, radiotherapy) may lead to remission of the myositis. Nevertheless most patients need extra immunosuppressive therapy (intravenous immunoglobulins, azathioprine, methotrexate, plasmapheresis, etc.) to control myositis and prevent relapses even if cancer remission is obtained. ${ }^{2,3,7}$

\section{Conclusion}

Paraneoplastic NAM is a rare syndrome. Diagnosis usually requires a muscle biopsy with typical aberrations.
Treatment with steroids and/or immunotherapy is suggested beside treatment of underlying neoplasm. Although disappearance of CAM has already been reported after surgery, radiotherapy or chemotherapy, we believe this is the first reported case of NAM resolving with an mTOR inhibitor in combination with steroids in a metastatic renal cell cancer patient. Further investigation is however needed to reveal the exact pathomechanism of NAM and thus to determine the exact role of on the one hand steroids and on the other hand mTOR inhibition in this case.

mTOR inhibition is currently investigated in other autoimmune disorders like rheumatoid arthritis, multiple sclerosis and hematological diseases. Specific data about treatment of NAM are however not yet available.

\section{References}

1 Hoogendijk JE, Amato AA, Lecky BR, Choy EH, Lundberg IE, Rose MR, et al. 119th ENMC international workshop: trial design in adult idiopathic inflammatory myopathies, with the exception of inclusion body myositis, 10-12 October 2003, Naarden, The Netherlands. Neuromuscul Disord. 2004;14:33745 .

2 Amato AA, Barohn RJ. Evaluation and treatment of inflammatory myopathies. J Neurol Neurosurg Psychiatry. 2009;80: $1060-8$.

3 Stenzel W, Goebel HH, Aronica E. Review: Immune-mediated necrotizing myopathies - a heterogeneous group of diseases with specific myopathological features. Neuropathol Appl Neurobiol. 2012;38:632-46.

4 Allenbach Y, Benveniste O. Acquired necrotizing myopathies. Curr Opin Neurol. 2013;26(5):554-60.

5 Kim HL, Belldegrun AS, Freitas DG, Bui MH, Han KR, Dorey FJ, et al. Paraneoplastic signs and symptoms of renal cell carcinoma: implications for prognosis. J Urol. 2003;170:1742-6.

6 Azar L, Khasnis A. Paraneoplastic rheumatological syndromes. Curr Opin Rheumatol. 2013;25:44-9.

7 Aggarwal R, Oddis CV. Paraneoplastic myalgias and myositis. Rheum Dis Clin N Am. 2011;37:607-21.

8 Dimachkie MM, Barohn RJ. Idiopathic inflammatory myopathies. Semin Neurol. 2012;32(3):227-36.

9 Casciola-Rosen L, Nagaraju K, Plotz P, Wang K, Levine S, Gabrielson E, et al. Enhanced autoantigen expression in regenerating muscle cells in idiopathic inflammatory myopathy. J Exp Med. 2005;201:591-601.

10 Andras C, Ponyi A, Constantin T, Csiki Z, Szekanecz E, Szodoray $\mathrm{P}$, et al. Dermatomyositis and polymyositis associated with malignancy: a 21-year retrospective study. J Rheumatol. 2008;35(3):438-44. 